\title{
Gender mainstreaming and sustainable development goals: a systematic literature review in post disaster reconstruction area
}

\author{
Tri Yumarni ${ }^{1, *}$, Dilanthi Amaratunga ${ }^{2}$ \\ ${ }^{1}$ Jenderal Soedirman University, Purwokerto Indonesia \\ ${ }^{2}$ Global Disaster Resilience Centre, University of Huddersfield UK
}

\begin{abstract}
This paper explores the linkage between gender mainstreaming and Sustainable Development Goals (SDGs) in the field of post-disaster reconstruction (PDR). A systematic review was conducted using Systematic Reviews and Meta-Analyses (PRISMA). We found a significant contribution of gender mainstreaming in achieving SDGs in the context of PDR. Some facilitators and barriers of mainstreaming gender into sustainable PDR were identified. Policy makers and implementers should aware those factors by reducing barriers while at the same time strengthening facilitators.
\end{abstract}

\section{Introduction}

The importance of gender mainstreaming for sustainable development is highlighted in the 2030 Sustainable Development Goals (SDGs). The full participation and equal involvement of women are needed to achieve the three pillars of SDGs: environmental sustainability, economic sustainability, and social sustainability.

To achieve environmental sustainability, it needs a strong knowledge about women and men related to natural or environmental resources and also their right and roles in source management and planning. Furthermore, it needs recognition and integration of both Women and men in understanding about environmental issues along with knowledge about the impact of environmental degradation and severe exploitation. In order to realize economic sustainability, it needs economic strategies which are gender responsive. Achievement of economic well-being will be difficult if there is still any group who is hugely living in poverty. To realize social sustainability, gender equality is vital to achieve. Hence, society is difficult to survive sustainably, or its people can live with dignity and confidence if there are prejudice and discrimination to any social group. This equality is an important pillar for developing resilience community.

While gender mainstreaming is recognized as an important concept in sustainable development theory and practice, the integration of this concept into thinking and practice of sustainable PDR is relatively new. In particular, a very little study in the area of PDR explores how women capacities should be integrated within policy arrangement and management within post-disaster recovery and reconstruction.

In this paper, we review the current literature on gender mainstreaming and sustainable PDR to answer the following questions: First, what are the linkages between gender mainstreaming and sustainable development goals in the context of PDR? Second, what are facilitators and barriers of mainstreaming gender within sustainable PDR?

\section{Material and method}

\subsection{Material}

A systematic literature review was used to collect the data. The literature on gender mainstreaming and sustainable PDR were retrieved from reliable sources particularly International journal and International organization report through Web of Science, Scopus, Google Scholars with keywords: gender mainstreaming, sustainable development and PDR.

\subsection{Method}

We applied PRISMA analysis by identifying, screening, and selecting relevant materials for the review. Figure 1 shows the PRISMA analysis conducted in this study.

We conducted the following steps in reviewing the materials. First, identifying relevant sources. From the Web of Science, Scopus and Google Scholar, we found 156 relevant journals, while from International organization report, we found 56 relevant published reports. Second, screening the source. We found 121 records after duplicates removed so that only 32 records we used for further analysis. Third, selecting the main sources. We found 12 main sources that the most relevant to answer the questions in the review.

* Corresponding author: triyumarni26@gmail.com 


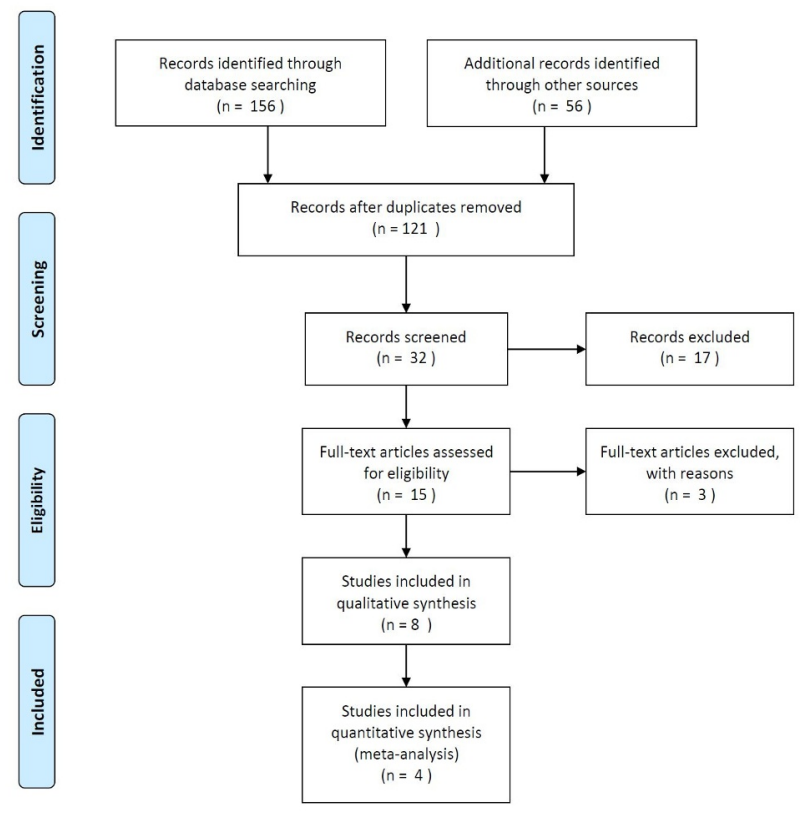

Fig. 1 PRISMA analysis applied in this study

\section{Results}

\subsection{Gender mainstreaming, sustainable PDR and SDGs}

ECOSOC defines gender mainstreaming as follow: "...the process of assessing the implications for women and men of any planned action, including legislation, policies or programs, in all areas and at all levels. It is a strategy for making women's as well as men's concerns and experiences an integral dimension of the design, implementation, monitoring, and evaluation of policies and programs in all political, economic and societal spheres so that women and men benefit equally and inequality is not perpetuated. The ultimate goal is to achieve gender equality." [1]

Gender mainstreaming is a means, not an end, to the gender equality goal. Thus, mainstreaming is a strategy to achieve gender equality [2]. It ensures gender perspectives and issues along with gender attention are central to development policy, programs, and projects. The mean of mainstreaming is not only put a "women" component or "gender equality" component into development policies and program and raising women's participation, but beyond those, it is also bringing women's and men's experience, knowledge, and interests to involve in the development activities and agenda [3]. By mainstreaming gender equality, there will be a transformation from an unequal situation of social and institutional structures into the same structure of social and institutional for women and men.

PDR activities include three main stages: planning, design, and construction [4]. Gender mainstreaming strategies can be integrated into each stage. Figure 2 presents the linkage of gender mainstreaming, PDR and three-pillars of SDGs.

The planning stage consists of activities in an identification of the overarching issue that must be acknowledged before decisions making whether and how to participate in reconstruction; which must be controlled and amend during the reconstruction implementation [4]. Crucial analysis in this stage is recognition of the disaster's context and its impact; acknowledgement of the district government structures; regulation and establishment of coordination methods; funding and timetable; selecting beneficiaries; building networks between stakeholders; identifying support means that is most appropriate; understanding natural hazards that threat in the future; and allocating resources [3]. The integration of gender mainstreaming strategies within planning stage includes the following activities: ensuring gender analysis within planning assessments, building objective on gender equality and women empowerment within planning and budgeting, awareness of gender vulnerabilities and concerns of different vulnerable groups of women (i.e. widows, girl children, and women with disabilities); and assess the different implications of planned programme for both women and men [5].

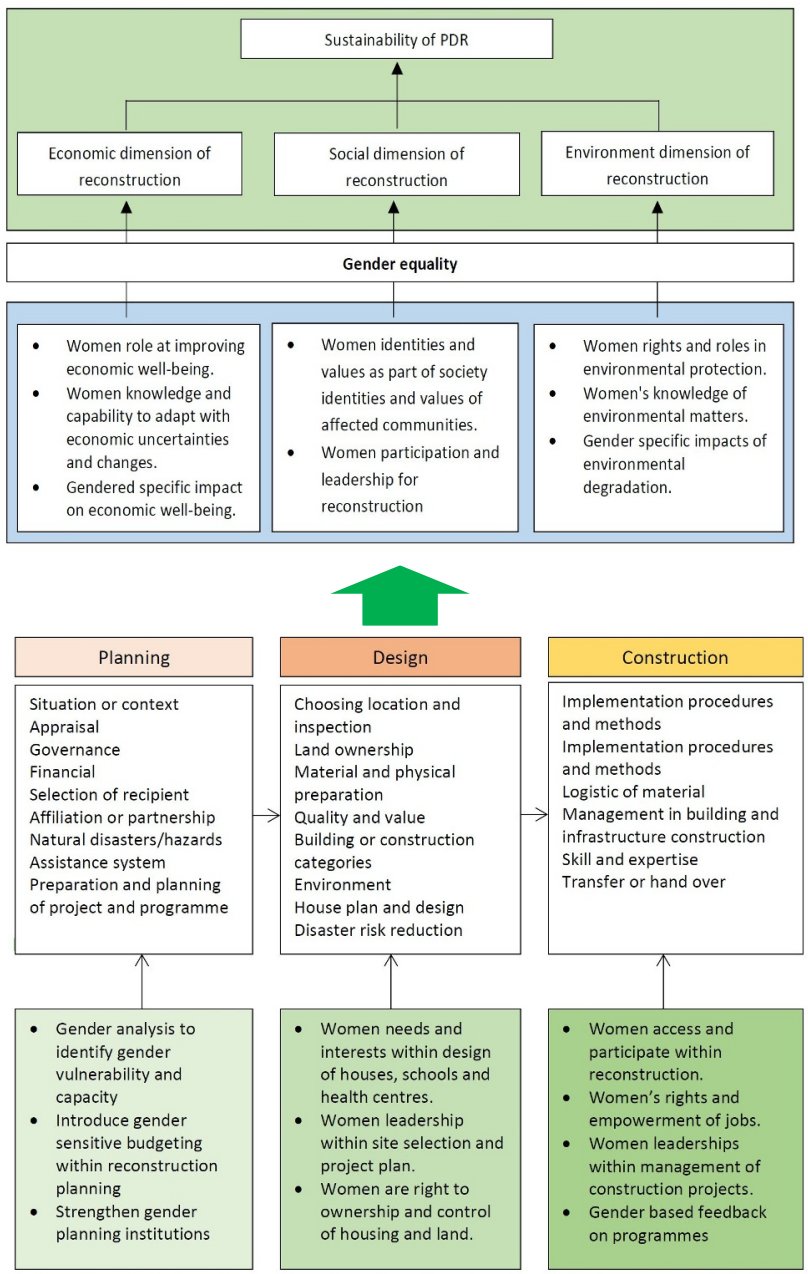

Fig. 2 The linkage between gender mainstreaming, PDR and three pillars of SDGs

Design step deals with the reconstruction project design in detail based on the previous decision to build temporary shelters or permanent housing [3]. The fundamental concerns related to this activity are including site selection; identification and resolve 
problems about land ownership; planning of physical settlement; identification of proper reconstruction quality; considering and integrating the strategy of disaster risk reduction; making design of the houses or shelters and other public buildings; planning the budget and break down it into detailed projects and activities [3]. The integration of gender mainstreaming strategies within design stage include ensuring women needs and interests reflected within a design of houses, schools, and health centers; women leadership within site selection and project plan; and support women's right to ownership and control of economic assets such as housing and land [5].

Moreover, the construction stage explains the execution of the reconstruction programs. It includes the considerations of implementation methods, project management of the construction, specification, procurement, and distribution of the reconstruction's material, labor and skill management, distribution of the buildings or handover, and evaluation along with maintenance of the buildings after the projects are completed [3]. The integration of gender mainstreaming strategies within construction stage include ensuring that women and men can access and to participate in reconstruction; work with men to secure their support for reconstruction activities that uphold women's rights and empowerment; involving women leadership within management of construction projects; ensure accountability by establishing mechanisms that enable both female and male beneficiaries to give feedback on reconstruction programs. It is also important that this stage includes feedback on the reconstruction program that particularly relates to gender issues [4].

The Sendai Framework for Disaster Risk Reduction and the 2030 Sustainable Development Agenda encourage all stakeholders to raise the issue about the role of both men and women in dealing with environmental problems to achieve sustainable development and to improve people quality life [6]. Achieving the three dimensions of sustainable development in the context of PDR can be done with investment in economic in an economic, social and environmental capital.

Environmental sustainability needs a good knowledge about the relation of women and environmental resources and also women's roles and right in managing and planning those resources. It will lead to the recognition of gender impact on environmental degradation as well as how to enhance sustainable environment after a disaster [6]. Hence, giving opportunities and resources as well as involving them in decision-making activities will enhance their well-being and social life that finally will give benefit for entire communities and moreover is going to improve the sustainability of reconstruction and development.

Economic sustainability needs strategies that responsive to the issue of gender. Women have a vital role in enhancing family and community well-being since the number of women in poverty following a disaster is increasing. Gendered impact on economic well-being should enhance economic sustainability of reconstruction [6]. If women are given access to and control over economic resources as well as opportunities to participate in all economic activities, they will fulfill the roles as operator of the reconstruction which result in better outcomes and benefits of sustainable economic development [6]. Moreover, it will contribute to economic growth as well as women's welfare.

Social sustainability concerns that reconstruction programs should identify the aspects of social values and identities, relationships and social organizations in disaster areas can be managed to adapt disasters in the future [7]. Women face obstacles to able to participate as a full and equal member in the reconstruction process, not to mention even in all aspects of life. Such obstacles are social norms, marginalization, gender stereotypes, low paid, limited access to and control over resources and health and education facilities. Hence, recognizing and integrating women identities and values is essential to maintain social identities and values of the affected communities. The active involvement and participation of women in PDR activities are needed, especially in identifying the concerns and needs of the disasteraffected communities.

In sum, the PDR process consists of some key considerations in which policymakers can include gender mainstreaming aspects within each process. It is critical to including gender mainstreaming elements on each process to create a gender-sensitive process of reconstruction [7]. However, effective implementation of gender mainstreaming within PDR also need to consider barriers and facilitators.

\subsection{Facilitators and barriers for implementing gender mainstreaming and sustainable PDR}

Moser and Moser present the most comprehensive review of gender mainstreaming and development across developing countries in the last decade [9]. On their review of gender mainstreaming and development practices conducted by international agencies (such as Hivos, DfID, Sida, Oxfam, World Bank, and UNDP), they elaborate two major conditions for effective gender mainstreaming for development in developing countries: (1) institutional input and (2) operational outcomes [10].

The institutional input means organizations and resources are supporting gender mainstreaming. These include a commitment to key actors, organization situation and culture, refusal and resistance, a mechanism for accountability, and gender training. Hence, gender mainstreaming success depends on key stakeholders' commitment along with their skill [11]. Many SDGs programs have better progress on genderrelated issues due to its gender specialists who work in the program [12]. There is a link between organizational culture and program success on gender mainstreaming to achieve gender equality, such as male-biased culture within the organization that related to attitudes, working conditions, structures, recruitment, and procedures, likely to discriminate against women both staff and clients [13].

On the other hand, operational outcomes refer to how to ensure the ultimate goals of gender mainstreaming is 
achieved. These constraining conditions include a lack of effective monitoring and evaluation system and women participation. The common problems within the operational stage include the lack of constant, effective, and organized monitoring and evaluation of the results and impacts of gender mainstreaming implementation. It leads to the difficulty in identifying the gender mainstreaming impacts on people live particularly on gender equality. It is important to link strategies to actual results. The important issue needs to be considered how to identify criteria for assessment with its indicators. Rather than concerning the impacts or outputs, an assessment most likely focuses on indicators of input like the number of women as beneficiaries, the female proportion of beneficiaries, and the number of genders mainstreaming activities [14].

Accordingly, the identification of gender indicators faces some issues and challenges. First, it needs the same or uniform criteria which are chosen by agreement and consensus. Second, it is hard to measure changes of status and transformation in power. Those challenges lead to a lengthy, costly and hard process in making impact assessment. Therefore, there is a significant issue related to the women participation that explaining about gender mainstreaming is not simply women participation, but more important is the situation and terms of the participation [14]. Some present examples bias said it was participation, but it was more mobilization of women that are often avoiding benefit for women [14].

In the contexts of PDR, studies identify some barriers for integrating gender mainstreaming. Firstly, policymakers often ignore the principles of participation and sustainability [14]. Policymakers often fail to realize that PDR is an intervention of development which must accommodate participation and sustainability. Short term PDR reflects technical respond that increases the involvement of experts from outside to assist the affected communities in skills, knowledge, and priorities. Accordingly, such a standardized and top-down policy and program from the government tend to ignore the complexity of affected communities needs, especially women need to rebuild their lives and well-being. Secondly, in term of participation within government, donor institutions, and international relief, there is often no apparent understanding about the form of women participation even though the urgency of women participation is widely recognized.

Thirdly, policy makers and implementers often fail to identify the gender-specific impact of the disaster. Increasing number of women household head and caregiving work following disasters lead to double burden among women in affected communities. PDR often ignores these issues, while it targets focuses on male-headed households for receiving reconstruction program [13]. Fourthly, a misconception is largely occurred by government and donors that women's groups are passive and based on traditional skills with low technology [14].

However, some studies also identify some facilitators for effective gender mainstreaming within PDR. Firstly, the reconstruction planning should be recognized women interests and make them as priority concerns [15]. Based on those women of different interest, planning must be able to address them and women concerns might be satisfied. Secondly, it is important to establish a strong framework of gender policy to integrate the gender objectives into sustainable PDR [13]. Moreover, applying a proactive and also systematic approach to gender integration within all reconstruction process such as assessment, design, implementation, monitoring, and evaluation, will likely give positive outcomes and develop a better balance in addressing problems, needs, and priorities for all beneficiaries, both men, and women. The method is used to design and implement a reconstruction program also influences the level of women contribution and participation in PDR activities. Women and women should be completely involved and consulted in every phase of design and implementation to make sure that the works are addressing their needs and circumstances. Thus, a training of gender-sensitive is often carried out as the first step or entry point for gaining understanding and acknowledgment of gender integration [14].

Thirdly, women participation and leadership are keys for success mainstreaming gender [12]. Hence, a reconstruction program should create a conducive environment for women and men to participate in the program. For example, providing more opportunities for female leaders and female staffs to involve in the program. Not only that, encouragement from local leaders so that women can participate in decision making is needed [13]. Educating gender mainstreaming for local leaders is needed so that they have knowledge and understanding of the importance of women participation and leadership in the reconstruction program.

Fourthly, the flexibility of reconstruction design which allows the reconstruction to adapt unique characteristics and lesson learn from the local community. Last but not least, mainstreaming gender during reconstruction program need more time and more fund. Therefore, adequate funding for all staffs and volunteers, as well as time for implementing every step of gender mainstreaming, are vital $[12,16]$. Gender mainstreaming is not only collecting genderdisaggregated data but most importantly how to increase women's participation through culturally-sensitive approaches.

\section{Conclusion}

This study highlight the vital contribution of gender mainstreaming in achieving sustainable development goals (SDGs) agendas in the context of post-disaster reconstruction. It is identified some facilitators and barriers for gender mainstreaming into sustainable postdisaster reconstruction. Effective gender mainstreaming strategies should recognize such conditions by creating and strengthening enabling conditions while minimizing constraining conditions. 


\section{References}

1. Aboobacker, N. P., and Nakray, K., Gender Mainstreaming in Disaster Management Policies: Indicators to Mitigate Vulnerability of Women. In Conference on Rethinking development in an Age of Scarcity and Uncertainty, New Values, Voices, and Alliances for Increased Resilience. University of York, UK (2011)

2. Byrne, B., and Baden, S., Gender, emergencies and humanitarian assistance (Institute of Development Studies, 1995)

3. Delaney, P. L., and Shrader, E., Gender and post-Disaster Reconstruction: The Case of Hurricane Mitch in Honduras and Nicaragua (The World Bank, Washington DC, 2000)

4. Enarson, E., Sex, Gender, and Gender Relations in Disasters (Sage publication private limited, India, 2009)

5. Enarson, E., and Meyreles, L., IJSSP 24, 10 (2004)

6. Fothergill, A., The International Journal of Mass Emergencies and Disasters (1996) 14 (1), 33

7. Ginige, K., Amaratunga, D., Haigh, R., DPM 18, 1, 23 (2009)

8. International Strategy for Disaster Reduction, Gender Mainstreaming in Disaster Reduction (UNISDR, Geneva, 2002)

9. Alston, M., Gender mainstreaming and climate change. In Women's Studies International Forum, 47, 1 (2004)

10. Bradshaw, S., Disasters 39, 1 (2015)

11. Gaillard, J. C. , Disasters 41, 3 (2017)

12. Schwarz, S., Critical perspectives on gender mainstreaming in disaster contexts. In Cultural Psychology of Coping with Disasters, Springer(2014), pp 323

13. Gaillard, J.C. Sexual and gender minorities in disaster. Gender \& Development 6,1 (2017)

14. Sohrabizadeh, S., PLoS currents 6, 1 (2014)

15. Juran, L., and Trivedi, J., Geographical Review 105, 4 (2015)

16. Morchain, D., Gender \& Development 23,3 (2015) 